\title{
Gossypiboma in a Child Presenting as Peri-Nephric Abscess
}

Sir,

Gossypiboma or retained surgical sponge (RSS) denotes a mass of cotton that is retained in the body following surgery. ${ }^{1}$ The true incidence is unknown, probably underreported because of medico-legal reasons.

A one-yr-11 month-old girl, presented with high fever and intermittent vomiting of $3 \mathrm{wk}$ duration with no localizing clues to the cause of fever. She was antenatally diagnosed to have right hydronephrosis and postnatally a duplication of right kidney with gross hydronephrosis of the dysplastic right upper moiety. She had recurrent UTIs till one yr of age and at one yr of age, had undergone a right hemi-nephro-ureterectomy elsewhere. Following surgery, she was well for about 10 months. She was febrile and toxic. Examination was normal except for tenderness in the right loin with a healed surgical scar in the right lumbar region.

Investigations revealed neutrophilic leukocytosis (TC 24600 cells $\left./ \mathrm{mm}^{3}\right)$, elevated CRP $(192 \mathrm{mg} / \mathrm{L})$, normal renal/liver parameters, sterile blood culture but urine culture grew significant E.coli (ESBL). USG abdomen showed fluid collection around right kidney and CT abdomen confirmed a right peri-nephric abscess. Incision and drainage (I \& D) done and about $150 \mathrm{ml}$ of pus drained which grew Salmonella typhi. Antibiotics were changed appropriately and she was discharged as the fever settled.

On follow up, she had persistent soaked dressing and discharge from I \& D site. USG abdomen showed hyperechoic area in upper pole of right kidney region. Repeat CT revealed a hypodense mass with peripheral enhancement in between the liver and lower pole of right kidney. (Fig. 1) Explorative laparotomy of that site revealed a RSS which was removed and the pus grew $S$. typhi with similar culture sensitivity pattern. Follow-up USG showed a collapsing cavity, the wound healed and she was asymptomatic.

RSS can cause 2 types of body responses: a) Aseptic fibrinous response with adhesions, encapsulation, granuloma formation, $b$ ) Exudative response \pm secondary bacterial contamination. The body attempts to extrude the foreign material either externally or into a hollow viscus, creating various fistulae. ${ }^{2,3,4}$ Our patient had the second type of response, who had undergone surgery at $1 \mathrm{yr}$ of age and the RSS remained asymptomatic for 10 months before presenting as peri-nephric abscess. The main complications include obstruction, peritonitis, adhesion, and abscess formation. Salmonella abscess complicating gossypiboma is unique. The probable explanation is a

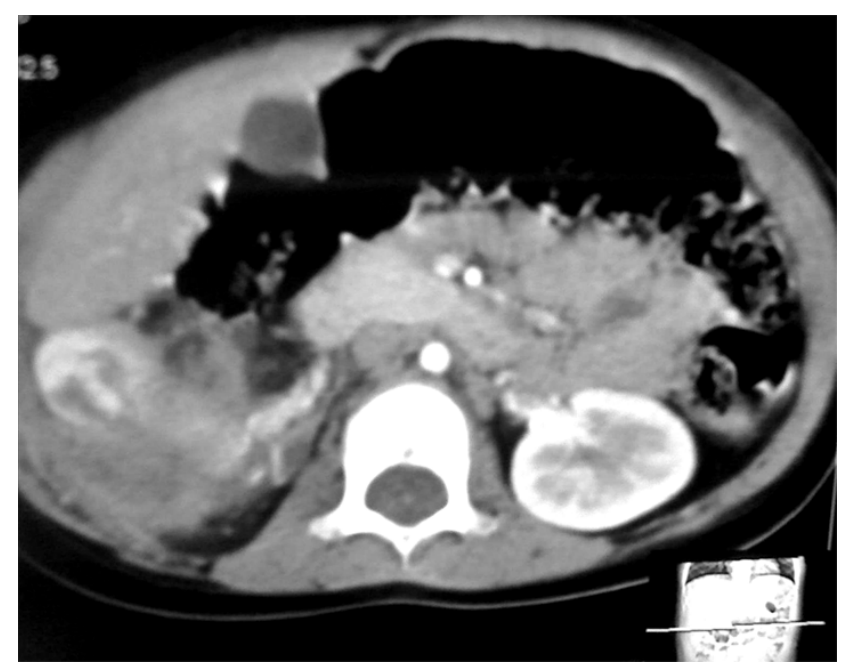

Fig. 1. Computerized tomography of abdomen showing the hypodense mass with peripheral enhancement between the liver and right kidney.

focal suppurative infection secondary to salmonella bacteremia and antibiotic resistance attributed to poor vascularity of RSS area. RSS should be the first differential in cases of unexplained fever or abscess in the operated area. Imaging studies are valuable tools to aid diagnosis. Radiography can detect sponges with radio-opaque marker. USG and CT are also valuable diagnosis tools.

It can be prevented by meticulous counting of all surgical materials pre and post operatively, thorough exploration of operative site before closure and use of sponges with radio-opaque marker.

S. Elayaraja, K. Kaarthigeyan, S. Sridharan ${ }^{1}$ and A. Andal

Department of Pediatrics and ${ }^{1}$ Department of Pediatric Surgery, Kanchi Kamakoti CHILDS Trust Hospital, Nungambakkam,

Chennai-34, TN, India

E-mail: kaarthigeyank@yahoo.com [DOI-10.1007/s12098-010-0010-8]

\section{REFERENCES}

1. Yildirim S, Tarim A, Nursal TZ, Yildirim T, Caliskan K, Torer $\mathrm{N}$ et al. Retained surgical sponge (gossypiboma) after intraabdominal or retroperitoneal surgery: 14 cases treated at a single center. Langenbecks Arch Surg 2006; 391: 390-395.

2. Hyslop JW, Maull KI. Natural history of the retained surgical sponge. South Med J 1982; 75: 657-660.

3. Rappaport W, Haynes K. The retained surgical sponge following intra-abdominal surgery. A continuing problem. Arch Surg 1990; 125: 405-407.

4. Kaiser CW, Friedman S, Spurling KP, Slowick T, Kaiser HA. The retained surgical sponge. Ann Surg 1996; 224: 79-84. 